\title{
of cigarette smoke on mouse lens through a multi OMIC approach
}

\begin{abstract}
Shahid Y. Khan ${ }^{1}$, Muhammad Ali ${ }^{1}$, Yura Jang ${ }^{2}$, Taekyung Ryu ${ }^{2}$, Andrew J. Schwab ${ }^{3}$, Brian O. Ingram ${ }^{3}$, Peter H. Cable ${ }^{4}$, Chan Hyun $\mathrm{Na}^{2}$, James T. Handa ${ }^{1}$ \& S. Amer Riazuddin ${ }^{1 \bowtie}$

Here, we report a multi OMIC (transcriptome, proteome, and metabolome) approach to investigate molecular changes in lens fiber cells (FC) of mice exposed to cigarette smoke (CS). Pregnant mice were placed in a whole-body smoke chamber and a few days later pups were born, which were exposed to CS for 5 hours/day, 5 days/week for a total of $31 / 2$ months. We examined the mice exposed to CS for CS-related cataractogenesis after completion of the CS exposure but no cataracts were observed. Lenses of CS-exposed and age-matched, untreated control mice were extracted and lens FC were subjected to multi OMIC profiling. We identified 348 genes, 130 proteins, and 14 metabolites exhibiting significant $(p<0.05)$ differential levels in lens FC of mice exposed to CS, corresponding to $3.6 \%, 4.3 \%$, and $5.0 \%$ of the total genes, protein, and metabolites, respectively identified in this study. Our multi OMIC approach confirmed that only a small fraction of the transcriptome, the proteome, and the metabolome was perturbed in the lens FC of mice exposed to CS, which suggests that exposure of CS had a minimal effect on the mouse lens. It is worth noting that while our results confirm that CS exposure does not have a substantial impact on the molecular landscape of the mouse lens FC, we cannot rule out that CS exposure for longer durations and/or in combination with other morbidities or environmental factors would have a more robust effect and/or result in cataractogenesis.
\end{abstract}

Cataract is a clouding or opacity of an otherwise transparent lens of the eye. Cataracts can be classified into the following two types: congenital cataracts caused by mutations in genes essential for development of the lens and maintenance of its transparency ${ }^{1-3}$, and age-related cataracts, a multifactorial disorder involving genetic susceptibility loci and environmental factors i.e., cigarette smoke (CS), and ultraviolet (UV) exposure, etc. ${ }^{4-6}$. Age-related cataract represents a significant burden of blindness worldwide that will grow as the age expectancy and population increases globally ${ }^{7}$.

CS has been identified as an important risk factor for development of cataracts ${ }^{8,9}$; however, the molecular mechanism of this association remains elusive. CS is a complex mixture of metal ions and different compounds responsible for the generation of reactive oxygen species (ROS $)^{10}$. Trace and heavy metals have been reported in rat lens exposed to tobacco smoke ${ }^{11}$. In a recent study $p$-benzoquinone induced changes are reported as a causative factor of CS-related cataractogenesis in guinea pig lens ${ }^{12}$.

The ocular lens consists of two, morphologically distinct, cell subpopulations: a monolayer of epithelial cells on the anterior side of the lens, and terminally differentiated fibers cells (FC) that account for the majority of lens volume ${ }^{13}$. Lens FC are generated throughout life by differentiation of lens epithelial cells; however, since lens FC are anuclear and organelle-free, they do not have the ability to repair, and therefore, damage to lens FC results in the opacification of an otherwise transparent lens ${ }^{13}$.

Here, we adopted a multi OMIC (transcriptome, proteome, and metabolome) approach to investigate molecular changes in lens FC of mice exposed to CS. We identified 348 genes, 130 proteins and 14 metabolites exhibiting significant $(\mathrm{p}<0.05)$ differential levels including diminished levels of the branched-chain amino acid (BCAA)related metabolites in lens FC of mice exposed to CS. To the best of our knowledge, this is the first report of a multi OMIC approach to investigate changes at a molecular level in lens FC of mice exposed to CS.

\footnotetext{
${ }^{1}$ The Wilmer Eye Institute, Johns Hopkins University School of Medicine, Baltimore, MD 21287, USA. ${ }^{2}$ Department of Neurology, Institute for Cell Engineering, Johns Hopkins University School of Medicine, Baltimore, MD 21205, USA. ${ }^{3}$ Metabolon Inc, Morrisville, NC 27560, USA. ${ }^{4}$ Department of Environmental Sciences and Engineering, University of North Carolina, Chapel Hill, NC 27599, USA. ${ }^{\varpi}$ email: riazuddin@jhmi.edu
} 

after completion of the CS exposure

T

The lenses of CS-exposed and untreated Ct mice were extracted, and FC
were separated from lens epithelium

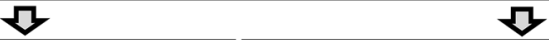

Transcriptome profiling ** four lens FC replicas of CS-exposed and Ct mice

FC replicas of CS-exposed

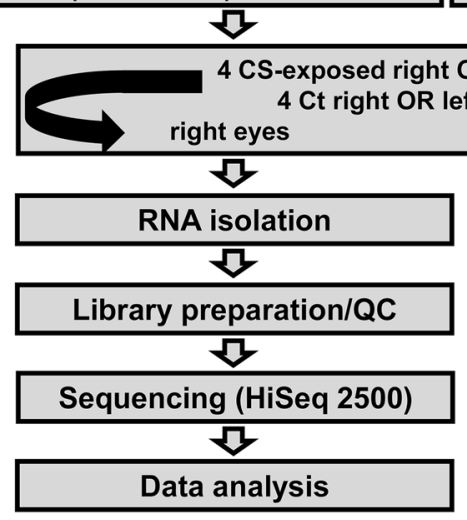

four lens FC replicas of CS-exposed and Ct mice
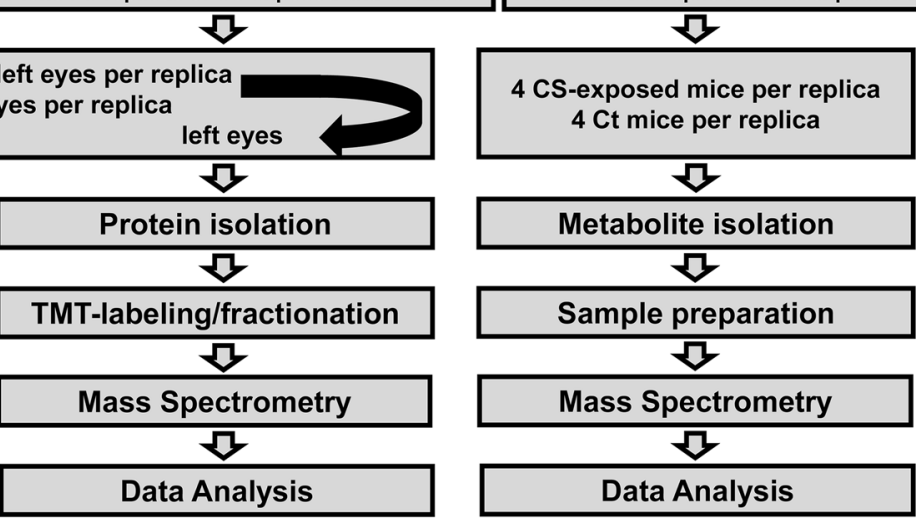

${ }^{* *}$ The four replicas of the CS-exposed and Ct mice consisted of lens FC from equal number of male and female mice.

Figure 1. Illustration of the work flow adopted to investigate the effects of cigarette smoke (CS) on mouse lens fiber cells (FC) through a multi OMIC (transcriptome, proteome, and metabolome) approach. A total of 32 newborn mice were exposed to CS in a smoke chamber for 5 hours/day, 5 days/week for a total of $3 \frac{1}{2}$ months (105 days). The mice exposed to CS exposure were examined for CS-related cataractogenesis. The CS-exposed and age-matched, untreated control $(\mathrm{Ct})$ mice were euthanized, lenses were extracted, lens FC were separated from the epithelium and used for next-generation RNA sequencing-based transcriptome, mass spectrometry-based proteome, and mass spectrometry-based metabolome profiling. The replicas of FC samples from both CS-exposed and untreated Ct mice consisted of lens from equal number of male and female mice.

\section{Results and discussion}

Here, we evaluate changes at a molecular level in lens FC of mice exposed to CS through a multi OMIC approach (Fig. 1). Pregnant mice (gestation days 18-20) were placed in a whole-body smoke chamber and 2-3 days later pups were born. A total of 32 pups (16 males and 16 females) along with four adult female mice were exposed to CS for 5 hours/day, 5 days/week for a total of $3 \frac{1}{2}$ months. In parallel, age-matched, four adult female mice and 32 ( 16 males and 16 females) pups were housed in the animal facility, hereafter referred to as untreated control (Ct) mice. We examined the mice exposed to CS for CS-related cataractogenesis after completion of the CS exposure but no cataracts were observed. The images of the lens of mice exposed to CS (Fig. 2, CS1-32) were similar to images of the lens of untreated Ct mice (Fig. 2, CS1-4). To investigate if mice exposed to CS develop cataracts over time, we examined the lens of the four adult female mice exposed to CS, 9-months post-exposure but no cataracts were observed in these mice (Fig. 2, CS33-36).

We performed next-generation RNA sequencing (RNA-Seq)-based transcriptome, mass spectrometrybased proteome, and mass spectrometry-based metabolome profiling of lens FC exposed to CS. The lenses were extracted from CS-exposed and untreated Ct mice and FC were separated from lens epithelium under a microscope. The extracted lens FC were maintained in distinct pools from either four right or four left eyes to serve as biological replicates. As illustrated in Fig. 1, the right eyes were used for transcriptome while the corresponding left eyes were used for proteome profiling. As $25 \mathrm{mg}$ of lens FC mass is required for the metabolome analysis, each biological replicate from either CS-exposed or untreated Ct mice consisted of lens FC from both eyes of four mice.

The next-generation sequencing resulted in $424.07 \times$ and $397.36 \times$ sequence coverage for lens FC of CSexposed and untreated Ct mice, respectively. The mapped reads were assembled into transcripts, and gene expression was measured and normalized using the fragment per kilobase per million mapped reads (FPKM) method, which identified the expression ( $\geq 1.0$ FPKM) of 9531 and 9590 genes in lens FC of CS-exposed and untreated Ct mice, respectively (Supplementary Data 1). The analysis identified 348 differentially expressed (DE) genes ( $\mathrm{p}<0.05)$, including 186 down- and 162 up-regulated genes in lens FC of mice exposed to CS (Supplementary Data 1). Moreover, an additional analysis was performed to determine the differential gene expression in lens FC of mice exposed to CS. The standard deviation (SD) was calculated for each transcript expression fold change $(\log 2)$ as a deviation from its mean of 0 or no change. The analysis revealed $332 \mathrm{DE}$ genes $(> \pm 2 \mathrm{SD})$, including 


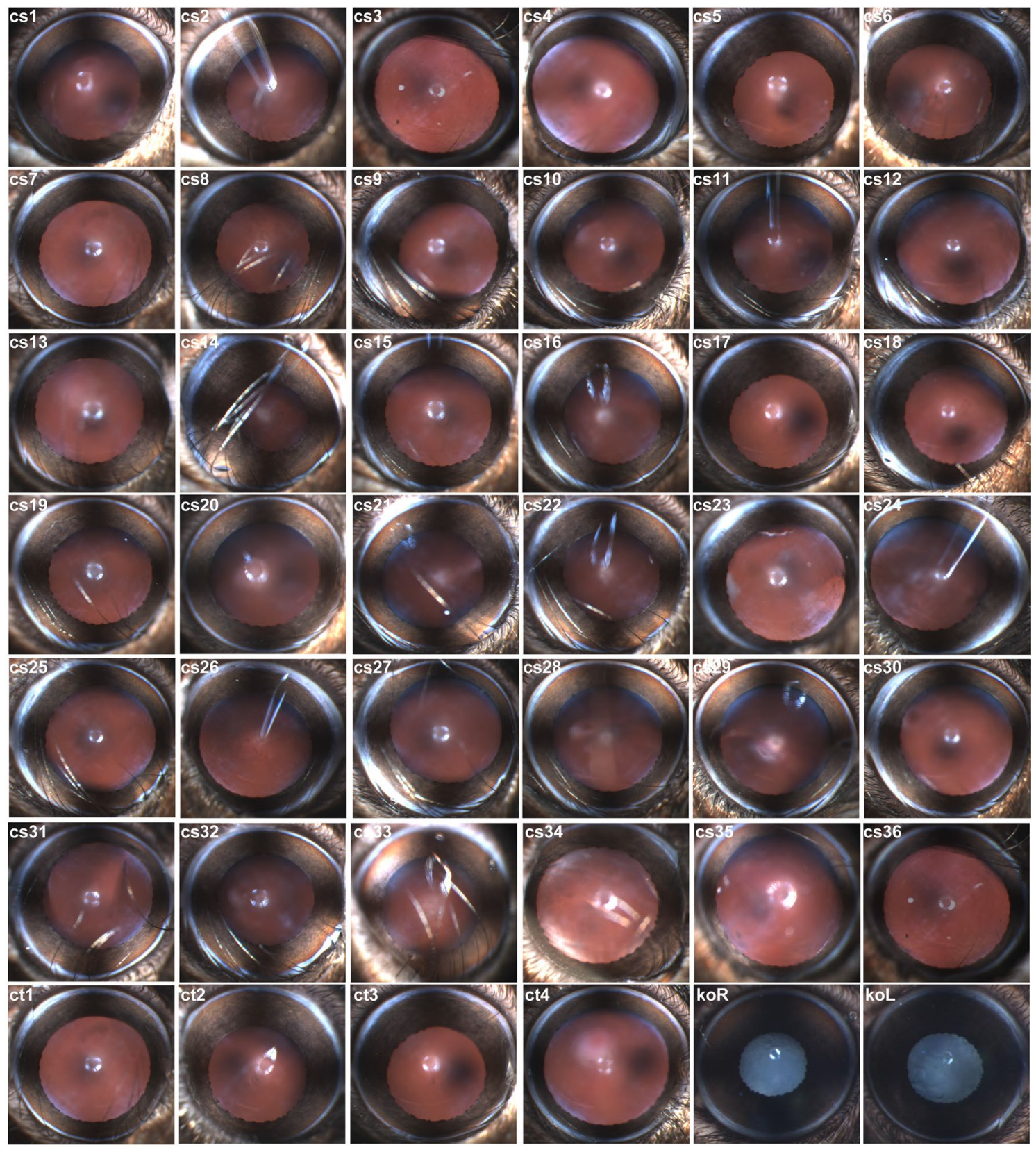

Figure 2. Evaluation of lens phenotype in cigarette smoke (CS)-exposed and age-matched, untreated control (Ct) mice. The 32 mice exposed to CS were examined for CS-related cataractogenesis after completion of $\mathrm{CS}$ exposure and age-matched untreated $\mathrm{Ct}$ mice but no cataracts were observed. The images of the lens of mice exposed to CS (CS1-32) were similar to images of the lens of age-matched, untreated Ct mice (Ct1-4). The four adult female mice exposed to CS were examined, 9-months post-exposure for development of cataracts but no cataracts were observed in these mice (CS33-36). The koR and koL illustrate the cataractous lens (right and left eyes, respectively) from a cataract-causing gene knockout mouse.

201 down- and 131 up-regulated genes in lens FC of mice exposed to CS (Fig. 3A). Col4a4, Col4a5, and Col6a3 were among the down-regulated genes in lens FC of mice exposed to CS (Supplementary Data 1). Mutations in Col4a4 and Col4a5 result in Alport syndrome along with developmental cataracts. Additionally, we identified the differential expression of Prmt3, Rarb, Caprin2, Gdi2, Oat, Herc1, and Agl in lens FC of mice exposed to CS (Supplementary Data 1). It is worth noting that loss of Prmt3, Rarb, Caprin2, Gdi2, Oat, Herc1, and Agl have been associated with abnormal lens FC morphology and cataracts in mice (http://www.informatics.jax.org/).

In parallel, mass spectrometry generated a total of 84,063 peptide spectrum matches (PSMs), yielding 23,882 total peptides, corresponding to 2323 proteins. We had previously reported 5404 proteins in the mouse lens proteome $^{14}$, and since the number of proteins identified here was significantly less (i.e., 2323 vs. 5404), we performed a second 8-plex TMT experiment to rule out the possibility of a technical error. We detected 2352 proteins in the second TMT experiment, a nearly similar protein count identified in the first TMT experiment. 

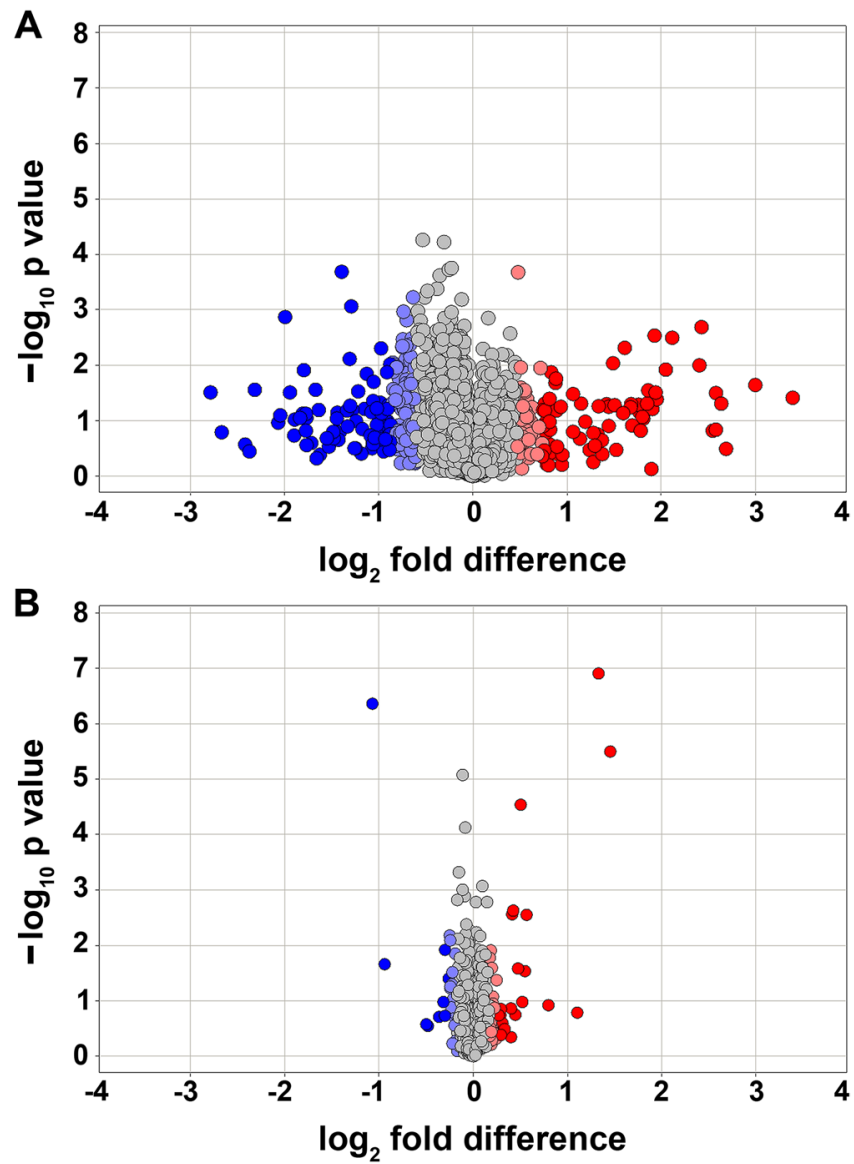

Figure 3. Illustration of genes and proteins perturbed in mouse lens fiber cells (FC) exposed to cigarette smoke (CS). (A) Volcano plot of differentially expressed (DE) genes in lens FC of mice exposed to CS. The transcriptome profiling identified $332 \mathrm{DE}$ genes $(> \pm 2 \mathrm{SD})$, including 201 down-regulated and 131 up-regulated in FC of mice exposed to CS. (B) Volcano plot illustrating differential protein levels in lens FC of mice exposed to CS. The proteome profiling identified 101 proteins ( $> \pm 2$ SD) including 59 exhibiting elevated levels and 42 displaying diminished levels in FC of mice exposed to CS. The fold changes are represented in the $\log 2$ scale depicted on the $\mathrm{x}$-axis, whereas the $-\log 10 \mathrm{p}$-value is depicted on the $\mathrm{y}$-axis (the use of -log values mean that genes/proteins with greater statistical significance are higher in the plot). The genes and proteins that are significantly elevated are highlighted in red and light red, and those with significantly diminished levels are highlighted in blue and light blue.

Perhaps, the age of the mice i.e., embryonic, and early postnatal lens compared to 3 months old mice lens and/ or the use of the whole lens (lens epithelium and FC used in previously reported study) compared to lens FC used in the current study may have contributed to the difference in protein counts.

The combined analysis of both proteome datasets identified a total of 130 proteins with differential levels $(\mathrm{p}<0.05)$, including 42 exhibiting elevated and 88 diminished levels in lens FC of mice exposed to CS (Supplementary Data 2). To further validate the DE protein, we reanalyzed the proteome datasets by calculating the SD for each protein fold change $(\log 2)$ as a deviation from its mean of 0 or no change. The analysis revealed 101 DE proteins $(> \pm 2 \mathrm{SD})$, including 59 exhibiting elevated and 42 diminished levels in lens FC of mice exposed to CS (Fig. 3B). We identified the elevated levels of CTNND2, a delta catenin protein in lens FC of mice exposed to CS (Supplementary Data 2) that has recently been associated with age-related cortical cataracts ${ }^{15}$. Additionally, we identified a decreased levels of CHORDC1, CRYGB, GCLC, HSD17B4, PANK4, PCBD1, OARD1, and HIP1R in lens FC of mice exposed to CS (Supplementary Data 2). Loss of CHORDC1, CRYGB, GCLC, HSD17B4, PANK4, PCBD1, OARD1, and HIP1R has been associated with abnormal lens morphology and cataracts in mice (http://www.informatics.jax.org/). The proteome also revealed diminished levels of GCLC, PCBD1, CRYGB, and PANK4 in lens FC of mice exposed to CS (Supplementary Data 2). Fan and colleagues recently identified nuclear cataracts in lens-specific Gclc knockout mice ${ }^{16}$. Bayle and colleagues reported hyperphenylalaninemia and cataracts in $P c b d 1$ knockout mice ${ }^{17}$. Liu et al. reported a point mutation in Crygb responsible for dominant nuclear cataracts in mice $^{18}$. Sun and colleagues reported a mutation in PANK4 liable for congenital posterior cataracts and importantly the Pank4 null mice develop cataracts ${ }^{19}$.

Next, we completed the mass spectrometry-based metabolome profiling of lens FC of mice exposed CS. We identified a total of 280 metabolites in lens FC of mice exposed to CS (Supplementary Data 3). Among the 280 
A

\begin{tabular}{|l|c|c|}
\hline \multicolumn{1}{|c|}{ Biochemical } & $\begin{array}{c}\text { CS-FC vs. Ct-FC } \\
\text { fold change }\end{array}$ & $\begin{array}{c}\text { CS-FC vs. Ct-FC } \\
\text { p value }\end{array}$ \\
\hline alpha-hydroxyisocaproate & 0.80 & 0.097 \\
\hline 1-carboxyethylleucine & 0.83 & 0.006 \\
\hline isoleucine & 0.83 & 0.021 \\
\hline leucine & 0.86 & 0.086 \\
\hline valine & 0.81 & 0.097 \\
\hline
\end{tabular}

B

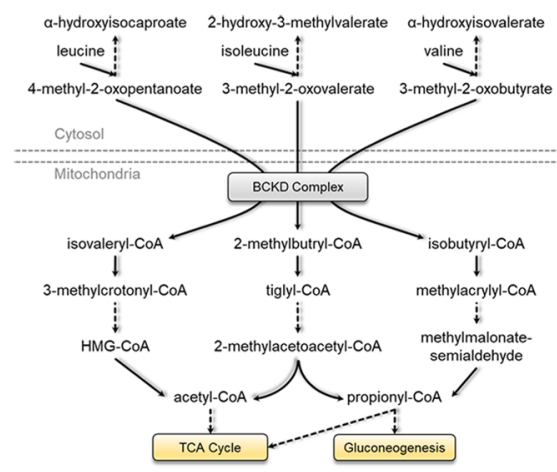

D

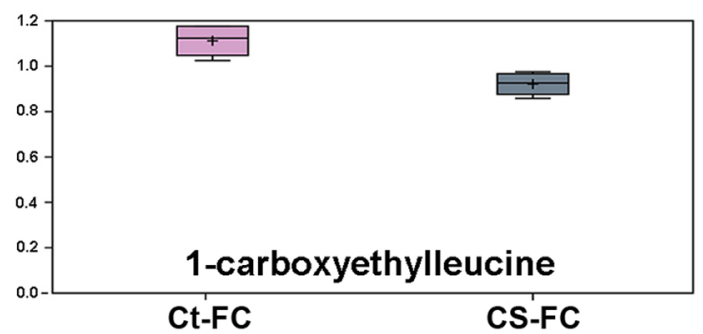

$\mathbf{F}$

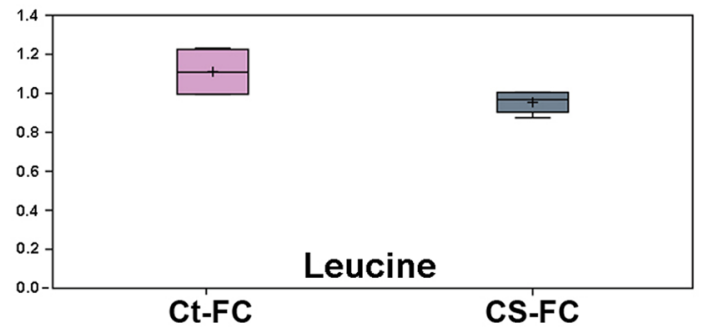

C

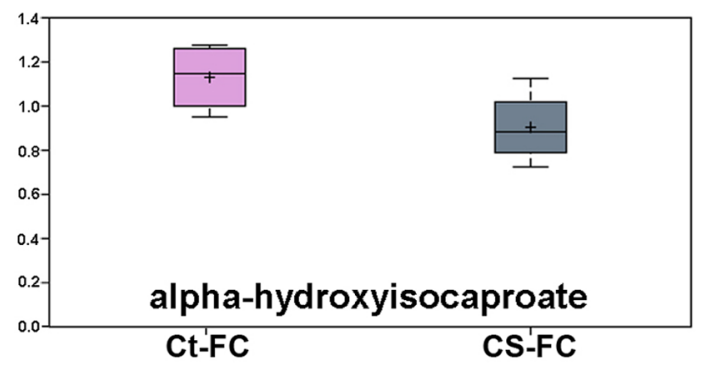

$\mathbf{E}$

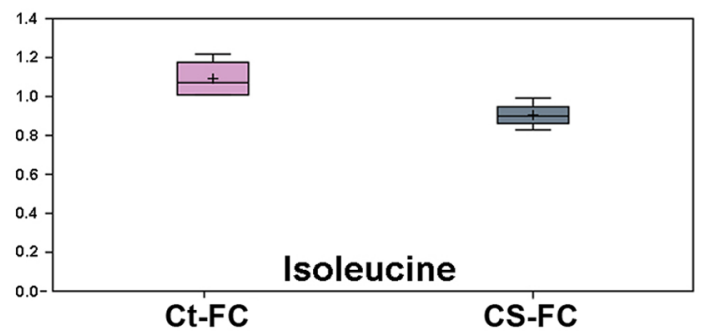

G

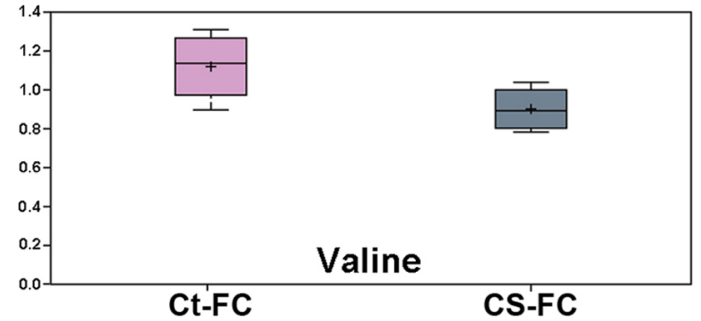

Figure 4. Metabolome analysis revealed diminished levels of branched-chain amino acids (BCAAs)-related metabolites in mouse lens fiber cells (FC) exposed to cigarette smoke (CS). (A) The diminished levels of BCAAs-related metabolites (alpha-hydroxyisocaproate, 1-carboxyethylleucine, isoleucine, leucine, and valine) identified in lens FC of mice exposed to CS. (B) Schematic of the pathway involving branched-chain a-keto acid dehydrogenase (BCKD), a multi-enzyme complex, which catalyzes BCAAs to the corresponding acylCoA derivatives. (C-G) Box plots depicting relative levels of BCAAs metabolites in lens FC of CS-exposed and untreated Ct mice. Diminished levels of (C) alpha-hydroxyisocaproate, (D) 1-carboxyethylleucine, (E) Isoleucine, (F) Leucine, and (G) Valine were identified in lens FC of mice exposed to CS.

metabolites identified in lens FC of mice exposed to CS, we detected the differential levels of 14 metabolites $(p<0.05)$, including eight metabolites exhibiting higher levels and six metabolites displaying lower levels (Supplementary Data 3). We identified a decrease in branched-chain amino acid (BCAA)-related metabolites in lens FC of mice exposed to CS (Fig. 4A) including isoleucine, leucine, and valine, which are essential amino acids with key roles in protein synthesis, energy metabolism, and cell signaling ${ }^{20}$.

The BCAA metabolites have reported being involved in oxidative metabolism and energy production through a catabolic pathway involving branched-chain $\alpha$-keto acid dehydrogenase (BCKD), a multi-enzyme complex,

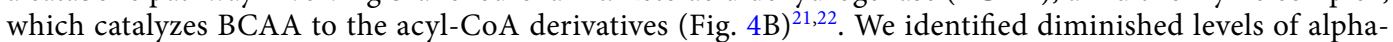
hydroxyisocaproate, 1-carboxyethylleucine, isoleucine, leucine, and valine metabolites in lens FC of mice exposed 


\begin{tabular}{|l|l|l|l|l|}
\hline \multirow{2}{*}{ Sample } & \multicolumn{2}{l}{ Cadmium } & \multicolumn{2}{l|}{ Nickel } \\
\cline { 2 - 5 } & Concentration $(\mathbf{p p b})$ & RSD & Concentration $(\mathbf{p p b})$ & RSD \\
\hline Ct-ml-1 & 0.253 & 26.0 & 12.150 & 10.2 \\
\hline Ct-ml-2 & 0.519 & 19.0 & 16.455 & 4.8 \\
\hline Ct-ml-3 & 0.196 & 19.5 & 9.977 & 7.7 \\
\hline Ct-ml-4 & 0.538 & 12.1 & 19.550 & 8.3 \\
\hline CS-ml-1 & 1.487 & 3.6 & 20.152 & 5.1 \\
\hline CS-ml-2 & 1.366 & 8.6 & 20.863 & 2.0 \\
\hline CS-ml-3 & 1.541 & 10.1 & 23.134 & 8.7 \\
\hline CS-ml-4 & 1.178 & 8.2 & 25.652 & 5.0 \\
\hline
\end{tabular}

Table 1. Mass spectrometry-based metal ions analysis of lungs from mice exposed to cigarette smoke (CS) and age-matched, untreated control mice. The quantification shows differential concentrations of cadmium $(\mathrm{Cd})$, and nickel (Ni) in the lungs of mice exposed to CS. The metal ion concentrations are normalized for sample mass and dilution. The metal ion concentrations are a mean value of five scans. RSD (relative standard deviation) is standard deviation divided by the mean value of the five scans (concentration in Table 1) and reported as a percentage. The detection limits were 0.2 , and $2.0 \mathrm{ppb}$ for $\mathrm{Cd}$, and $\mathrm{Ni}$ respectively. $\mathrm{Ct}$ - $\mathrm{ml}$ control mouse lungs, $C S$ - $m l$ cigarette smoke-exposed mouse lungs, $p p b$ parts per billion (also equilment to ng/g).

to CS (Fig. 4C-G). In a recent study, Tomoda and colleagues analyzed the levels of BCAA-related metabolites and identified a significant decreased in BCAA levels in both plasma and skeletal muscles of rats exposed to $\mathrm{CS}^{23}$. We further detected a decrease of 1-carboxyethylleucine in lens FC of mice exposed to CS. Importantly, Das et al. reported the role of leucine and its transporters in protection against CS-induced cell death in the lungs ${ }^{21}$.

Finally, we searched for the metabolome data to identify metabolites that are trending in the same direction i.e., elevated and/or diminished levels in lens FC of mice exposed to CS but are not statistically significant. The analysis revealed elevated levels of twenty-eight metabolites associated with long-chain fatty acids, lysophospholipids, monoacylglycerols, and endocannabinoids in lens FC of mice exposed to CS (Supplementary Table 1). In parallel, decreased levels of 20 metabolites associated with phosphatidylethanolamine (PE), phosphatidylserine (PS), pentose metabolism, Fructose, Mannose, and Galactose Metabolism, and nucleotide Sugar in lens FC of mice exposed to CS (Supplementary Table 2). The low counts of sugar metabolism-related metabolites are inline with the low levels of BCAA related metabolites, involved in oxidative metabolism and energy production ${ }^{22}$.

Since we observed a minimal effect of CS exposure on the molecular landscape of lens FC with no cataracts in mice exposed to CS, we sought evidence of CS exposure by examining Cadmium (Cd), and Nickel (Ni) in the lungs of mice exposed to CS. The premise of the experiment was based on the results of two independent studies reporting a 3.1- and 1.7-fold increase in Cd concentration in the serum of tobacco cigarette smokers ${ }^{24,25}$. The premise was also based on the identification of $\mathrm{Cd}$ and $\mathrm{Ni}$ in mainstream particulates of cigarettes containing tobacco ${ }^{26}$.

We performed total metal quantification in lungs of mice exposed to CS using an Agilent 7500cx inductively coupled plasma mass spectrometer. The analysis identified a 3.70-fold $(p=0.0001)$ and a 1.54-fold $(p=0.01)$ higher concentration of $\mathrm{Cd}$ and $\mathrm{Ni}$, respectively in the lungs of mice exposed to $\mathrm{CS}$ compared with untreated $\mathrm{Ct}$ mice (Table 1 and Supplementary Table 3 ). The statistically significantly higher concentrations of Cd and Ni in the lungs of mice exposed to CS are in line with chronic exposure to CS.

In summary, we report results of a multi OMIC approach to examine the molecular landscape of mice lens FC exposed to CS for $3 \frac{1}{2}$ months, which confirmed a minimal effect on the molecular landscape of lens FC. However, we cannot rule out that CS exposure for longer durations (i.e., $>3 \frac{1}{2} 2$ months) and/or in combination with other morbidities or environmental factors would have a more robust effect and/or result in cataractogenesis.

\section{Materials and methods}

Animals included in the study. The use of mice in this study was approved by the Johns Hopkins Animal Care and Use Committee (ACUC; Baltimore, MD, USA), and all experiments were performed in accordance with the approved protocol by the Institutional Review Board (IRB) of the Johns Hopkins University School of Medicine (Baltimore, MD) and consistent with the Association of Research in Vision and Ophthalmology (ARVO) statement for the use of animals in ophthalmic and vision research. The C57BL/6J mice strain (Stock \# 000664; Jackson laboratory) was used for all experiments.

The study design included placing pregnant mice (gestation days 18-20) in a whole-body smoke chamber and a few days later pups were born. Of these, four adult females along with 32 pups (16 males and 16 females) were exposed to CS for 5 hours/day, 5 days/week, and remained in the chamber for a total of $3 \frac{1 / 2}{2}$ months (105 days). In parallel, age-matched, four adult female mice and 32 (16 males and 16 females) pups were housed in the animal facility for $3 \frac{1}{2}$ months that served as untreated controls.

Exposure to CS in a whole-body smoke chamber. The mice were exposed to CS as described ${ }^{27,28}$. The smoke chamber contained a smoking machine (TE-10, Teague Enterprises, Davis, CA) that burns five cigarettes (2R4F reference cigarettes ( $2.45 \mathrm{mg}$ nicotine/cigarette; Tobacco Research Institute, University of Kentucky) at a time, taking two-second duration puffs at a flow rate of $1.05 \mathrm{l} / \mathrm{min}$, to provide a standard puff of $35 \mathrm{~cm}^{3}$, providing a total of eight puffs per minute. The machine was adjusted to produce sidestream (89\%) and mainstream 
smoke (11\%). The chamber atmosphere was monitored to maintain total suspended particulate at $90 \mathrm{mg} / \mathrm{m}^{3}$ and carbon monoxide at $350 \mathrm{ppm}$.

Evaluation of lens phenotype in CS-exposed and untreated Ct mice. The mice were examined for CS-related cataractogenesis after completion of CS exposure ${ }^{29}$. The eyes of CS-exposed and untreated CT mice were dilated using tropicamide (1\%) and phenylephrine (2.5\%) followed by anesthetization by ketamine/ xylazine (100 mg/kg body weight for ketamine and $16 \mathrm{mg} / \mathrm{kg}$ body weight for xylazine). The lenses were examined with a slit-lamp microscope and images were taken by Phoenix Micron III Retinal Imaging Microscope (Phoenix Research Labs, Pleasanton, CA, USA).

Extraction of the lenses and separation of lens FC. The mice were anesthetized with isoflurane and euthanized through cervical dislocation. The lenses were extracted from CS-exposed and untreated Ct mice and FC were separated from the lens epithelium using forceps under a microscope. The lens FC from CS-exposed and untreated $\mathrm{Ct}$ mice was maintained at $-80^{\circ} \mathrm{C}$ in distinct pools (i.e., biological replicates), each consisting of FC of the right eye or the left eye.

We used four biological replicates (two male and two female) of the CS-exposed and untreated Ct mice, each consisting of lens FC of the right eyes for transcriptome profiling and their respective left eyes for proteome profiling. As a minimum of $25 \mathrm{mg}$ of lens FC mass was required for metabolome analysis, each of the four biological replicates of the CS-exposed and untreated Ct mice consisted of eight lens FC (both right and left eyes) from four male or four female mice.

Transcriptome profiling. Next generation RNA-Seq of lens FC of CS-exposed and untreated Ct mice was performed commercially by Novogene Corporation Inc. (Sacramento, CA). A total of four biological replicates, each consisting of pooled lens FC of the right eyes of four CS-exposed and untreated Ct mice was used for transcriptome profiling and the RNA-Seq data were analyzed as described ${ }^{29}$.

The FPKM expression values were imported in the Spotfire DecisionSite with Functional Genomics (TIBCO Spotfire, Boston, MA) software for further evaluation and graphical representation. All transcripts $\log 2$-fold changes between lens FC of CS-exposed and untreated Ct mice were analyzed to determine the SD from their mean of 0 , which represents no change.

Proteome profiling. Four biological replicates, each consisting of pooled lens FC of the left eyes of four CSexposed mice and four untreated Ct mice were used for proteome profiling. A total of $200 \mu \mathrm{g}$ of protein for each sample was used for each replicate. The reduction and alkylation of the proteins were conducted with $10 \mathrm{mM}$ of tris (2-Carboxyethyl) phosphine hydrochloride and $40 \mathrm{mM}$ of chloroacetamide for $1 \mathrm{~h}$ at room temperature. Mass spectrometry-based proteome profiling was completed as described ${ }^{14}$.

The abundance values of reporter ion intensities from the 8-plex TMT experiment were imported into Partek Genomics Suite v6.6 (Partek, Inc., St. Louis, MO, USA) for protein annotation and differential expression analysis. The normalized reporter ion intensities were examined for SD to investigate the differential expression in lens FC of mice exposed to CS compared with lens FC of untreated Ct mice. The p values were estimated by a two-tailed t-test, assuming a hypothesized mean of 0 change. The normalized ratios were converted to a $\log _{2}$ scale (becoming the conventional "log-ratios" or "log2 fold changes") for statistical and graphic representation.

Metabolome profiling. Four biological replicates, each consisting of pooled lens FC, wet mass (25 mg) of four CS-exposed and untreated Ct mice eyes (right and left) were used for metabolome profiling. The FC were frozen immediately at $-80^{\circ} \mathrm{C}$ until further processing for metabolome profiling. Metabolome profiling and data analysis were performed commercially by Metabolon, Inc. (Morrisville, NC, USA) as described ${ }^{30}$.

Sample preparation involved metabolite extraction with methanol and the resulting extracts were analyzed on an accurate mass global metabolomics platform consisting of multiple arms differing by chromatography method and mass spectrometry ionization mode ${ }^{30,31}$. Metabolites were identified by automated comparison of the ion features in the experimental samples to a reference library of chemical standard using software developed at Metabolon ${ }^{32,33}$.

Metal ion analysis. Metal ion quantification and data analysis were performed by the Biomarker Mass Spectrometry Facility (UNC-Chapel Hill, NC). A total of four biological replicates, each consisting of pooled mice lungs wet mass (250-460 mg) of four CS-exposed and untreated Ct mice were used for metal ion analysis. Mouse lungs were frozen immediately at $-80^{\circ} \mathrm{C}$ upon extraction, until further processing. The samples were digested with a combination of concentrated nitric acid, $30 \%$ hydrogen peroxide, and heat. The digested samples were diluted to $4 \mathrm{ml}$ with deionized water. Quantification of total Cd, and Ni, was performed using Agilent $7500 \mathrm{cx}$ inductively coupled plasma mass spectrometer (ICP-MS; Santa Clara, CA) as described ${ }^{34}$.

ARRIVE guidelines. This study was completed in compliance with the ARRIVE guidelines.

\section{Data availability}

RNA-Seq raw reads and processed data of lens FC of CS-exposed and untreated Ct mice have been deposited in the NCBI Gene Expression Omnibus and are accessible through the GEO accession number GSE144818. The mass spectrometry data of lens FC of CS-exposed and untreated Ct mice have been deposited to the ProteomeXchange Consortium via the PRIDE partner repository with the dataset identifier PXD017414 and PXD020421. 
The metabolome raw data of lens FC of CS-exposed and untreated Ct mice have been deposited in the MetaboLights repository and are accessible through study identifier MTBLS207.

Received: 30 December 2020; Accepted: 14 July 2021

Published online: 22 September 2021

\section{References}

1. Foster, A. \& Johnson, G. J. Magnitude and causes of blindness in the developing world. Int. Ophthalmol. 14, 135-140 (1990).

2. McCarty, C. A. \& Taylor, H. R. The genetics of cataract. Invest Ophthalmol. Vis. Sci 42, 1677-1678 (2001).

3. Pascolini, D. \& Mariotti, S. P. Global estimates of visual impairment: 2010. Br. J. Ophthalmol 96, 614-618 (2012).

4. West, S., Munoz, B., Emmett, E. A. \& Taylor, H. R. Cigarette smoking and risk of nuclear cataracts. Arch. Ophthalmol 107, 1166-1169. https://doi.org/10.1001/archopht.1989.01070020232031 (1989).

5. Wu, R. et al. Smoking, socioeconomic factors, and age-related cataract: The Singapore Malay Eye study. Arch. Ophthalmol 128, 1029-1035.https://doi.org/10.1001/archophthalmol.2010.147 (2010).

6. Ye, J. et al. Smoking and risk of age-related cataract: A meta-analysis. Invest Ophthalmol. Vis. Sci 53, 3885-3895. https://doi.org/ 10.1167/iovs.12-9820 (2012).

7. Asbell, P. A. et al. Age-related cataract. Lancet 365, 599-609. https://doi.org/10.1016/S0140-6736(05)17911-2 (2005).

8. Hiller, R. et al. Cigarette smoking and the risk of development of lens opacities. The Framingham studies. Arch. Ophthalmol. 115, 1113-1118. https://doi.org/10.1001/archopht.1997.01100160283003 (1997).

9. Cumming, R. G. \& Mitchell, P. Alcohol, smoking, and cataracts: The Blue Mountains Eye Study. Arch. Ophthalmol. 115, 1296-1303. https://doi.org/10.1001/archopht.1997.01100160466015 (1997).

10. Shalini, V. K. et al. Oxidative damage to the eye lens caused by cigarette smoke and fuel smoke condensates. Indian J. Biochem. Biophys 31, 261-266 (1994).

11. Avunduk, A. M., Yardimci, S., Avunduk, M. C., Kurnaz, L. \& Kockar, M. C. Determinations of some trace and heavy metals in rat lenses after tobacco smoke exposure and their relationships to lens injury. Exp. Eye Res. 65, 417-423. https://doi.org/10.1006/exer. 1997.0344 (1997).

12. Chowdhury, A. et al. p-Benzoquinone-induced aggregation and perturbation of structure and chaperone function of alphacrystallin is a causative factor of cigarette smoke-related cataractogenesis. Toxicology 394, 11-18. https://doi.org/10.1016/j.tox. 2017.11.018 (2018).

13. Lovicu, F. J. \& McAvoy, J. W. Growth factor regulation of lens development. Dev. Biol. 280(1), 1-14. https://doi.org/10.1016/j.ydbio. 2005.01.020 (2005).

14. Khan, S. Y. et al. Proteome profiling of developing murine lens through mass spectrometry. Invest. Ophthalmol. Vis. Sci. 59, $100-107$. https://doi.org/10.1167/iovs.17-21601 (2018).

15. Jun, G. et al. Delta-Catenin is genetically and biologically associated with cortical cataract and future Alzheimer-related structural and functional brain changes. PLoS ONE 7, e43728. https://doi.org/10.1371/journal.pone.0043728 (2012).

16. Fan, X. et al. The LEGSKO mouse: A mouse model of age-related nuclear cataract based on genetic suppression of lens glutathione synthesis. PLoS ONE 7, e50832. https://doi.org/10.1371/journal.pone.0050832 (2012).

17. Bayle, J. H. et al. Hyperphenylalaninemia and impaired glucose tolerance in mice lacking the bifunctional DCoH gene. J. Biol. Chem 277, 28884-28891. https://doi.org/10.1074/jbc.M201983200 (2002).

18. Liu, H. et al. Crystallin \{gamma\}B-I4F mutant protein binds to \{alpha\}-crystallin and affects lens transparency. J. Biol. Chem. 280, 25071-25078. https://doi.org/10.1074/jbc.M502490200 (2005).

19. Sun, M. et al. A novel mutation of PANK4 causes autosomal dominant congenital posterior cataract. Hum. Mutat. 40, 380-391. https://doi.org/10.1002/humu.23696 (2019).

20. Jewell, J. L., Russell, R. C. \& Guan, K. L. Amino acid signalling upstream of mTOR. Nat. Rev. Mol. Cell Biol. 14, 133-139. https:// doi.org/10.1038/nrm3522 (2013).

21. Das, B. et al. Leucine and its transporter provide protection against cigarette smoke-induced cell death: A potential therapy for emphysema. Toxicol. Rep. 1, 752-763. https://doi.org/10.1016/j.toxrep.2014.09.011 (2014).

22. Yeaman, S. J. The 2-oxo acid dehydrogenase complexes: Recent advances. Biochem. J. 257, 625-632. https://doi.org/10.1042/bj257 0625 (1989).

23. Tomoda, K. et al. Branched-chain amino acid-rich diet improves skeletal muscle wasting caused by cigarette smoke in rats. J. Toxicol. Sci. 39, 331-337. https://doi.org/10.2131/jts.39.331 (2014).

24. Barany, E. et al. Trace element levels in whole blood and serum from Swedish adolescents. Sci. Total Environ. 286, 129-141. https:// doi.org/10.1016/s0048-9697(01)00970-6 (2002).

25. Satarug, S. \& Moore, M. R. Adverse health effects of chronic exposure to low-level cadmium in foodstuffs and cigarette smoke. Environ. Health Perspect. 112, 1099-1103. https://doi.org/10.1289/ehp.6751 (2004).

26. Bache, C. A., Lisk, D. J., Doss, G. J., Hoffmann, D. \& Adams, J. D. Cadmium and nickel in mainstream particulates of cigarettes containing tobacco grown on a low-cadmium soil-sludge mixture. J. Toxicol. Environ. Health 16, 547-552. https://doi.org/10.1080/ 15287398509530762 (1985).

27. Ali, M. et al. Cigarette smoke triggers loss of corneal endothelial cells and disruption of descemet's membrane proteins in mice. Invest. Ophthalmol. Vis. Sci. 62, 3. https://doi.org/10.1167/iovs.62.3.3 (2021).

28. Fujihara, M., Nagai, N., Sussan, T. E., Biswal, S. \& Handa, J. T. Chronic cigarette smoke causes oxidative damage and apoptosis to retinal pigmented epithelial cells in mice. PLOS ONE 3, e3119. https://doi.org/10.1371/journal.pone.0003119 (2008).

29. Khan, S. Y. et al. Transcriptome profiling of developing murine lens through RNA sequencing. Invest. Ophthalmol. Vis. Sci. 56, 4919-4926. https://doi.org/10.1167/iovs.14-16253 (2015)

30. Khan, S. Y., Ali, M. \& Riazuddin, S. A. Metabolome profiling of the developing murine lens. Exp. Eye Res. 108343. https://doi.org/ 10.1016/j.exer.2020.108343 (2020).

31. Ford, L. et al. Precision of a clinical metabolomics profiling platform for use in the identification of inborn errors of metabolism. J. Appl. Lab. Med. 5, 342-356. https://doi.org/10.1093/jalm/jfz026 (2020).

32. DeHaven, C. D., Evans, A. M., Dai, H. \& Lawton, K. A. Organization of GC/MS and LC/MS metabolomics data into chemical libraries. J. Cheminform. 2, 9. https://doi.org/10.1186/1758-2946-2-9 (2010).

33. Evans, A. M., DeHaven, C. D., Barrett, T., Mitchell, M. \& Milgram, E. Integrated, nontargeted ultrahigh performance liquid chromatography/electrospray ionization tandem mass spectrometry platform for the identification and relative quantification of the small-molecule complement of biological systems. Anal. Chem. 81, 6656-6667. https://doi.org/10.1021/ac901536h (2009).

34. Laine, J. E. et al. Placental cadmium levels are associated with increased preeclampsia risk. PLoS ONE 10, e0139341. https://doi. org/10.1371/journal.pone.0139341 (2015). 


\section{Acknowledgements}

This study was supported by the National Eye Institute Grants R01EY022714 (SAR), R01EY027691 (JTH), and the Robert Bond Welch Professorship (JTH). We acknowledge the support of the NIH shared instrumentation grant S10OD021844. The Biomarker Mass Spectrometry Facility, UNC Environmental Science and Engineering is partially funded by CEHS NIH Grant P30-ES10126 provided metal analysis. The authors acknowledge Sonny Dike for managing the smoke chamber and Marisol Cano for the management of the satellite animal facility.

\section{Author contributions}

S.Y.K., M.A., and S.A.R.: conceived and designed the experiments; C.H.N., J.T.H., and S.A.R.: contributed reagents, materials, and analytical tools; S.Y.K., M.A., Y.J., T.R., A.J.S., B.O.I., P.H.C., and C.H.N.: performed experiments; S.Y.K., M.A., Y.J., T.R., A.J.S., B.O.I., P.H.C., C.H.N., and S.A.R.: analyzed the data; S.Y.K., M.A., Y.J., T.R., A.J.S., B.O.I., P.H.C., C.H.N., J.T.H., and S.A.R.: contributed to writing the manuscript.

\section{Competing interests}

The authors declare no competing interests.

\section{Additional information}

Supplementary Information The online version contains supplementary material available at https://doi.org/ 10.1038/s41598-021-95013-7.

Correspondence and requests for materials should be addressed to S.A.R.

Reprints and permissions information is available at www.nature.com/reprints.

Publisher's note Springer Nature remains neutral with regard to jurisdictional claims in published maps and institutional affiliations.

(c) Open Access This article is licensed under a Creative Commons Attribution 4.0 International License, which permits use, sharing, adaptation, distribution and reproduction in any medium or format, as long as you give appropriate credit to the original author(s) and the source, provide a link to the Creative Commons licence, and indicate if changes were made. The images or other third party material in this article are included in the article's Creative Commons licence, unless indicated otherwise in a credit line to the material. If material is not included in the article's Creative Commons licence and your intended use is not permitted by statutory regulation or exceeds the permitted use, you will need to obtain permission directly from the copyright holder. To view a copy of this licence, visit http://creativecommons.org/licenses/by/4.0/.

(C) The Author(s) 2021 